\title{
Neurotensin Receptor 1 (NTSR1) Overexpression in Breast Carcinomas Is Common and Independent of ER/PR/Her2 Expression
}

\author{
Xianyong Gui ${ }^{1 *}$, Shuhong Liu ${ }^{1}$, Ziran Meng ${ }^{1}$, Zu-Hua Gao ${ }^{2}$ \\ ${ }^{1}$ Calgary Laboratory Services, Department of Pathology and Laboratory Medicine, University of Calgary, Calgary, Canada; \\ ${ }^{2}$ Department of Pathology, McGill University, Montreal, Canada. \\ Email: ${ }^{*} x$ sean.gui@cls.ab.ca
}

Received May $5^{\text {th }}, 2013$; revised June $6^{\text {th }}, 2013$; accepted June $14^{\text {th }}, 2013$

Copyright (C) 2013 Xianyong Gui et al. This is an open access article distributed under the Creative Commons Attribution License, which permits unrestricted use, distribution, and reproduction in any medium, provided the original work is properly cited.

\begin{abstract}
Neurotensin (NT) is a 13-amino acid peptide with trophic effects on some neoplasms. Its bioactivities are mainly mediated by neurotensin receptor 1 (NTSR1). Both NT and NTSR1 were found to be upregulated in breast cancer. NT/NTSR1 thus becomes a potential therapeutic target. We studied whether any correlation exists between the expression of NTSR1 in breast carcinomas and the expression of ER, PR, and Her2. A total 85 cases of invasive ductal (62) and lobular (23) breast carcinomas were studied. Based on their ER/PR profiles, the ductal carcinomas (DCs) were subcategorized into $\mathrm{ER}+\mathrm{PR}+(21), \mathrm{ER}+/ \mathrm{PR}-(20)$, and $\mathrm{ER}-/ \mathrm{PR}-(21)$. All of the lobular carcinomas (LCs) were $\mathrm{ER}+/ \mathrm{PR}+.21 .57 \%$ of all DCs and $5.56 \%$ of LCs were Her2 positive. $77.78 \%$ of ER-/PR- DCs were also Her2 negative (triple negative). The expression of NTSR1 was detected by immunohistochemistry and was semiquantitated (as negative, $1+, 2+, 3+$ ). Both $2+$ and $3+$ were collectively defined as overexpression. The expression of NTSR1 was weak and focal in non-neoplastic mammary epithelial cells. It is increased in $74.19 \%$ of DCs $(80.95 \%$ in $\mathrm{ER}+\mathrm{PR}+, 75 \%$ in $\mathrm{ER}+/ \mathrm{PR}-$, and $66.67 \%$ in ER-/PR- group), and in $95.65 \%$ of LCs. The overexpression of NTSR1 is similar between ER+ DCs and ER- DCs $(75 \%$ vs $66.67 \%, p>0.05)$ as well as between PR+ DCs and PR- DCs $(80.95 \%$ in ER+/PR+ DCs vs $75 \%$ in ER+/PR-DCs, $p>0.05$ ). And it was seen in $77.78 \%$ of Her $2+$ DCs, $78.38 \%$ of Her $2-$ DCs, $94.12 \%$ of Her2- LCs, and $78.57 \%$ of triple negative DCs. Overall, NTSR1 is commonly overexpressed in both ductal and lobular breast carcinomas and is independent of the ER/PR/Her2 profiles of the tumors. The present data supports the potential benefit of developing NTSR1 blockers in the adjuvant therapy of breast carcinomas, particularly for those "triple negative" tumors.
\end{abstract}

Keywords: Neurotensin; Neurotensin Receptor; NTSR1; Breast Carcinoma; Estrogen Receptor; Progesterone Receptor; Her2

\section{Introduction}

Neurotensin (NT) is a 13-amino acid regulatory peptide that was initially isolated from bovine hypothalamus by Carraway and Leeman [1] and was subsequently found to express highest in the small intestine [2]. Its physiologic secretion is stimulated significantly by fat intake [3]. Over the years, NT was found to possess diverse functions including trophic effects on some normal and neoplastic cells. Most of NT's effects are mediated via highaffinity neurotensin receptor 1 (NTSR1), a member of G-protein-coupled receptor family. The activation of NT/NTSR1 pathway involves several intracellular sig-

${ }^{*}$ Corresponding author. naling events, including increased intracellular calcium release, activation of phosphatidylinositol 3'-kinase (PI3K), mitogen activating protein kinase (MAPK) family member ERK1/2, NF- $\kappa$ B pathway, and Rho family of small GTPases, leading to cell proliferation. The growthpromoting effect of NT/NTSR1 has been found in a variety of carcinomas including those of the colon, pancreas, prostate, and lung [4]. It has also been found in breast carcinomas that near all breast ductal carcinomas over express NTSR1 and about a third coexpress both NT (autocrine) and NTSR1 [5], NT exerted an inhibitory activity on the apoptosis in breast cancer cells [6], and the upregulated expression of NTSR1 is related to the tumor progression [7]. All of these features make NT/ 
NTSR1 an ideal novel supplementary therapeutic target in the hormonal therapy of breast cancer. Meanwhile, many efforts on development of NTSR1 antagonists (e.g., SR48692, SR142948) have been undertaken [8,9]. Additionally, NT/NTSR1 activation was found to stimulate the expression of epidermal growth factor receptor (EGFR) as well as to transactivate EGFR [10-12]. This is very interesting in view of that a member of the EGFR family, Her2 (cErbB2) is currently the primary hormonal therapeutic target for breast cancer. Moreover, a cross talk between NT and estrogen and between NT and progesterone has been noted, at least in the physiologic regulation of central nervous system [13-16]. In order to better evaluate the possible application and benefits of the anti-NTSR1 treatment, further understanding on whether the NTSR1 expression correlates with the expression of ER/PR/Her2, as well as the histologic type and grade of breast cancer will be important. Little data regarding these questions is currently available. In the present study we have found that NTSR1 overexpression is common in breast carcinomas and it is independent of the expression of ER, PR, or Her2.

\section{Methods and Materials}

\subsection{Breast Carcinoma Patients and Samples}

Eighty five cases of invasive breast carcinomas (age 24 83 , mean 59.98 years old), including both ductal (62) and lobular (23) types, resected by mastectomy or segmental resection were retrieved from the 2010 surgical pathology file at Calgary Laboratory Services. All tumors were graded histologically using the modified Bloom-Richardson (MBR) system.

The study was approved by the Research Committee of Calgary Laboratory Services. The ethics approval was granted by Calgary Health Research Ethics Board.

\subsection{Detection of NTSR1, ER, PR, and Her2}

One of the most representative sections of tumor was selected from each case for the study. The immunoreactivity of NTSR1 was detected by immunohistochemistry carried out on deparaffinized sections using the avidinbiotin-peroxidase complex method. The formalin fixed paraffin embedded tissue sections were pretreated in CINTec Epitope Retrieval Solution $(10 \mathrm{mM}$ Tris/1mM EDTA, pH 9.0) for 20 minutes at $95^{\circ} \mathrm{C}-100^{\circ} \mathrm{C}$, and then cooled to room temperature. The NTSR1 antibody was a rabbit polyclonal antibody (IMGENEX, California, USA) against the third cytoplasmic domain of human NTSR1. All slides were stained with Ventana Nexes IHC autostainer at 1:40 dilution using UltraView Universal DAB Detection (Ventana \#760 - 500). The expression of NTSR1 was semiquantitated based on the positivity and intensity of the immunostain as negative (no stain at all), $1+$ (faint or less than $10 \%$ of tumor cells stained), $2+$ (moderate intensity or up to $50 \%$ of tumor cells stained), and $3+$ (strong intensity and more than $50 \%$ of tumor cells stained). A comparison of NTSR1 expressions between these groups was analyzed. Both $2+$ and $3+$ were collectively defined as increased expression (overexpression) in our results, considering that $1+$ was observed in non-neoplastic ductal epithelial cells.

ER and PR expression were routinely assessed by immunohistochemistry (performed at the diagnostic immunohistochemistry laboratory of Calgary Laboratory Services), and Her2 was routinely detected by silver in-situ hybridization (SISH) (performed at the diagnostic laboratory of the Tom Baker Cancer Centre). The methodology of all of the lab tests and the result analysis were validated. The results were reported routinely and included in the clinical pathology reports.

\subsection{Statistic Analysis}

The rate (\%) of NTSR1 overexpression in tumors of different groups was calculated and compared between groups by Fisher Exact test.

\section{Results}

The details of the patients' information are summarized in Table 1.

Based on their ER/PR profiles, the ductal carcinomas (DCs) were subcategorized into $\mathrm{ER}+/ \mathrm{PR}+(21$ cases), $\mathrm{ER}+/ \mathrm{PR}-(20$ cases$)$, and ER-/PR- (21 cases). The 23 lobular carcinomas (LCs) were all ER+/PR+. The data of Her2 was available in 69 cases. 11 of $51(21.57 \%)$ DCs and 1 of 18 LCs $(5.56 \%)$ were Her2 positive. 14 of 18 (77.78\%) ER-/PR- DCs were also Her2 negative (triple negative).

The immunoreactivity of NTSR1 appeared in both membranous and cytoplasmic patterns, as shown in Figure 1. It was weak and partial in the non-neoplastic mammary epithelial cells, but it was stronger in both insitu and invasive carcinomas.

As shown in Figure 2, the overexpression of NTSR1 was detected in $46 / 62(74.19 \%)$ DCs $(80.95 \%$ in ER+l $\mathrm{PR}+, 75 \%$ in $\mathrm{ER}+/ \mathrm{PR}-$, and $66.67 \%$ in $\mathrm{ER}-/ \mathrm{PR}-$ group), and $22 / 23(95.65 \%)$ LCs. The overexpression of NTSR 1 appeared to be slightly elevated in tumors with the expression of both ER and PR, although no statistically significant difference existed $(p>0.05)$ between any of these groups based on our sample size.

As shown in Figure 3, the overexpression of NTSR1 was slightly more common in ER+ DCs than in ER- DCs (75\% versus $66.67 \%, p>0.05)$, and was similar between $\mathrm{PR}+\mathrm{DCs}$ and $\mathrm{PR}-\mathrm{DCs}(80.95 \%$ in $\mathrm{ER}+\mathrm{PR}+\mathrm{DCs}$ versus 
Table 1. Summary of breast cancer patients and NTSR1 immunohistochemistry.

\begin{tabular}{|c|c|c|c|c|c|c|c|c|c|c|c|}
\hline \multirow{2}{*}{ Type } & \multirow{2}{*}{ ER/PR } & \multirow{2}{*}{ Number } & \multirow{2}{*}{$\begin{array}{c}\text { Age } \\
\text { (mean) }\end{array}$} & \multicolumn{3}{|c|}{ MBR Grade } & \multirow{2}{*}{ Her2 (+/Total) } & \multicolumn{4}{|c|}{ NTSR1 } \\
\hline & & & & 1 & 2 & 3 & & Neg & $1+$ & $2+$ & $3+$ \\
\hline \multirow{3}{*}{ Ductal } & $\mathrm{ER}+/ \mathrm{PR}+$ & 21 & 63.67 & 6 & 11 & 4 & $4 / 16$ & 0 & 4 & 13 & 4 \\
\hline & $\mathrm{ER}+/ \mathrm{PR}-$ & 20 & 59.12 & 5 & 8 & 7 & $7 / 17$ & 0 & 5 & 4 & 11 \\
\hline & $\mathrm{ER}-/ \mathrm{PR}-$ & 21 & 60.91 & 0 & 3 & 18 & $4 / 18$ & 1 & 6 & 7 & 7 \\
\hline Lobular & $\mathrm{ER}+/ \mathrm{PR}+$ & 23 & 60.18 & 2 & 19 & 2 & $1 / 18$ & 0 & 1 & 19 & 3 \\
\hline
\end{tabular}

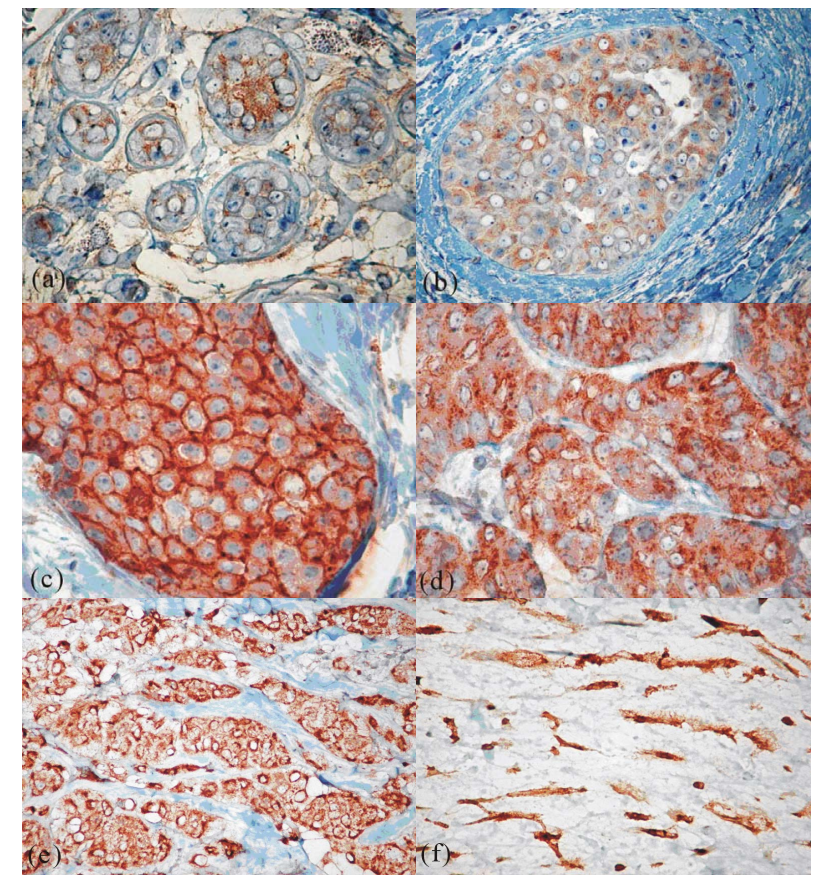

Figure 1. The intensity and pattern of NTSR1 expression on immunohistochemistry. (a) Weak (1+) expression in normal ductal epithelial cells $(\times 200)$; (b) Moderate expression $(2+)$ in ductal carcinoma in situ (DCIS) $(\times 200)$; (c) Relatively membranous pattern of immunostain $(\times 400)$; (d) Relatively cytoplasmic pattern of immunostain $(\times 400)$; (e) Strong expression $(3+)$ in invasive ductal carcinoma $(\times 200)$; (f) Strong expression (3+) in invasive lobular carcinoma (×200).

$75 \%$ in $\mathrm{ER}+/ \mathrm{PR}-\mathrm{DCs}, \mathrm{p}>0.05)$.

As shown in Figure 4, the overexpression of NTSR1 was seen in $7 / 9(77.78 \%)$ Her2+ DCs, 29/37 (78.38\%) Her2- DCs, and in 16/17 (94.12\%) Her2- LCs. In addition, the NTSR1 overexpression was seen in 11/14 (78.57\%) triple negative DCs (not shown in the figure). No statistically significant difference existed $(p>0.05)$ between any of these groups.

Between the tumors with different histologic grades, as shown in Figure 5, the expression of NTSR1 seemed to be negatively correlated with the histologic grade of the tumors. The difference between Grade 1 and Grade 3 was statistically significant $(\mathrm{p}=0.032)$, although the difference between Grade 1 and Grade 2, or between grade 2 and grade 3 , was not statistically significant.

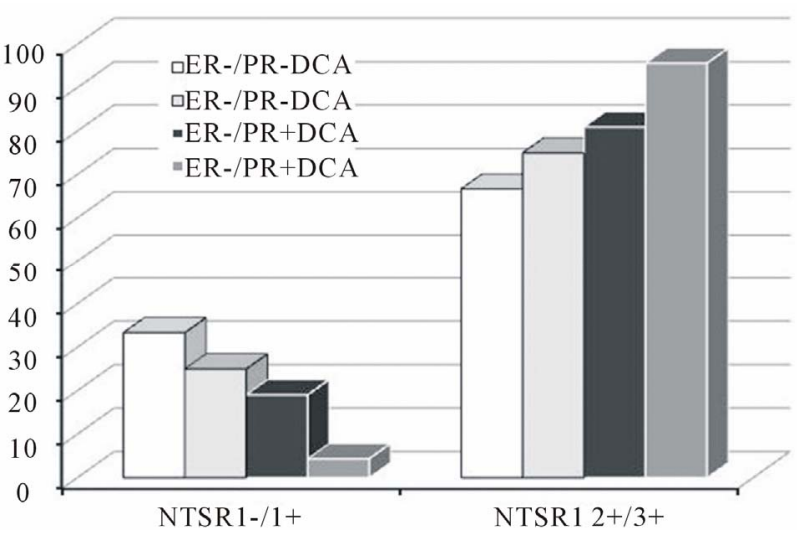

Figure 2. NTSR1 expression (\% of high expression) in different groups of breast carcinomas. No significant difference existed between any of the groups.

In summary, the overexpression of NTSR1 is less common in high grade carcinomas than in low grade ones. There seems to be a trend of higher expression of NTSR1 in ER+/PR+ tumors than in ER-/PR- tumors. However, there is no statistically significant difference between $\mathrm{ER}+$ and $\mathrm{ER}-, \mathrm{PR}+$ and $\mathrm{PR}-$, and between Her2+ and Her2- tumors.

\section{Discussion}

The detection of certain biologic markers in breast cancer has had significant clinical impact and become routine in the clinical pathologic assessment of breast carcinomas. These markers are related either to the aggressiveness, invasiveness, and the extent of tumor spread, or to the response of certain therapeutic agents. Thus they have both prognostic and predictive values. So far, the most useful biomarkers include estrogen receptor (ER), progesterone receptor (PR), Her2, and Ki67. Currently, the application of ER, PR, and Her2 in the selection and prediction of hormonal therapy in breast cancers is critical. The antagonists of ER (e.g., Tamoxifen) and Her2 (e.g., Trastuzumab) play important roles in the management of breast cancer patients. Several new markers have been explored in recent years, including p53, ARF, TBX2/3, Cyclin D1, Cyclin E, BRCA1, BRCA2, and VEGF [17]. The utility of these emerging novel markers are being investigated.

NT/NTSR1 is still a relatively little-known marker 


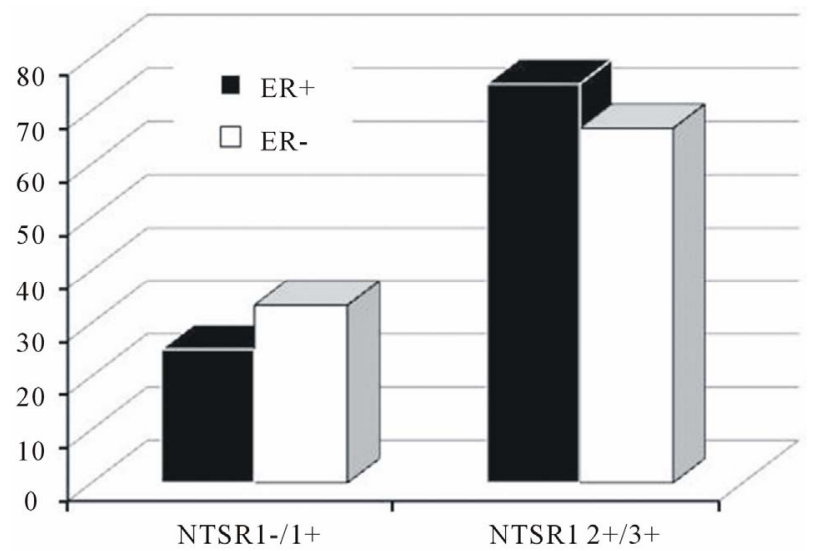

(a)

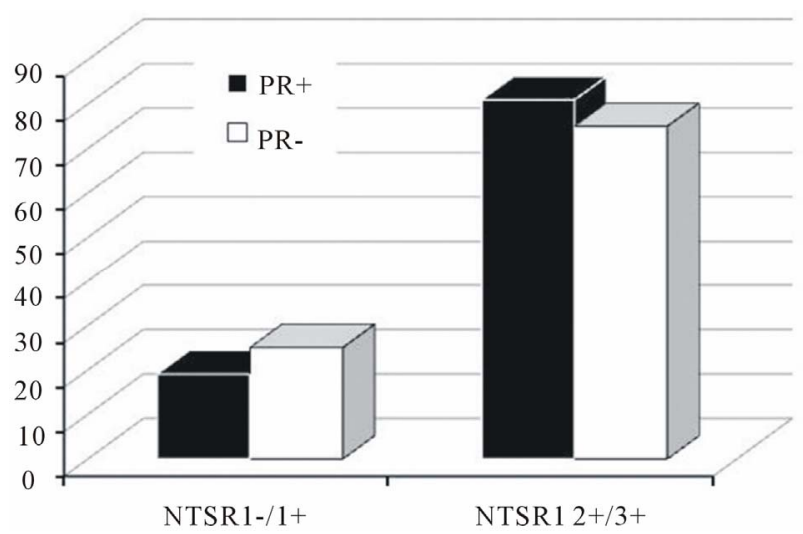

(b)

Figure 3. NTSR1 expression (\% of high expression) in breast carcinomas with regards to ER and PR expression. (a) Relationship of NTSR1 and ER expression; (b) Relationship of NTSR1 and PR expression. No significant difference existed between ER+ and ER- tumors or between PR+ and PR- tumors.

with regards to breast cancer. Although the tumor growthpromoting effects of NT/NTSR1 on breast cancer cells and the overexpression of NTSR1 in breast cancer tissue have been reported for years, the prognostic and predictive values of detection of NTSR1 in breast cancer have not been studied. In a study reported by Dupouy et al., the overexpression of NTSR1 seemed to be associated with the histological grade, size of tumor, and number of positive axillary lymph nodes, and it was also found to be an independent prognostic factor associated with the poor outcome [6]. In our data, however, the NTSR1 overexpression appeared to be negatively correlated with the histologic grade of the tumors. Further study is required to clarify this discrepancy.

In the present study, NTSR1 was found to be universally expressed in both ductal and lobular breast carcinomas, and was independent of the ER/PR/Her2-neu profiles of the tumors. The study was not designed to further corre-

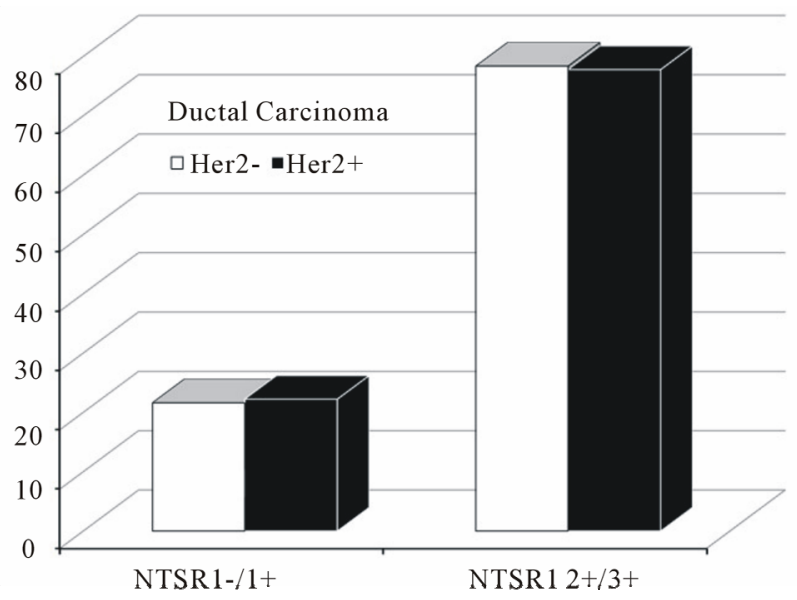

(a)

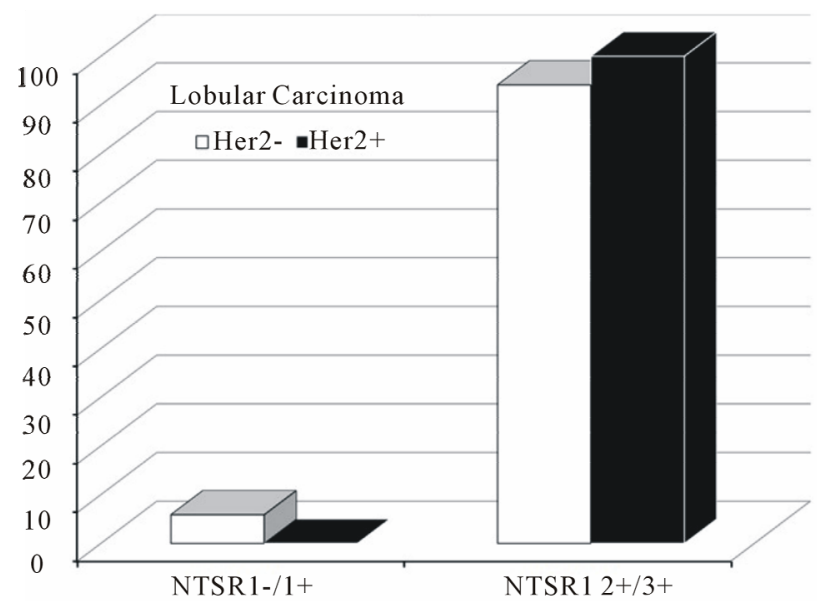

(b)

Figure 4. NTSR1 expression (\% of high expression) in breast carcinomas with regards to Her2 expression. No significant difference existed between Her2+ and Her2tumors. (a) Her2+ vs Her2- ductal carcinomas; (b) Her2+ vs Her2- lobular carcinoma.

late the NTSR1 expression with the patient outcome and many other cliniopathologic features. The data of the potentially prognostic value of NTSR1, unfortunately, is not available.

Our finding that NTSR1 is expressed in all kinds of breast carcinomas further supports the potential benefits of developing NTSR 1 blockers in the adjuvant therapy of breast carcinomas, particularly for the "triple negative" tumors. It is reasonable to postulate that the antiNT/NTSR1 approach would be a supplement and/or alternative to the established hormonal treatment of breast carcinomas. In addition, in view of the transactivation of NT/NTSR1 pathway on EGFR and the interaction be tween NT and estrogen/progesterone, the intervention of NT/NTSR pathway may also modulate the therapeutic effects of ER-/Her2 antagonists.

Several experimental studies using breast cancer cell 


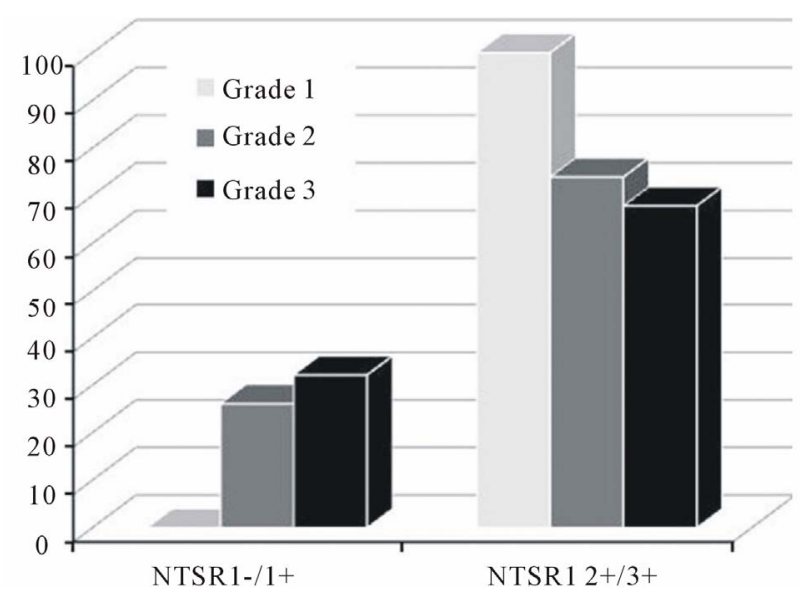

Figure 5. NTSR1 expression (\% of high expression) in breast carcinomas with regards to the histologic grade (MBR grading system). A significant difference existed between Grade 1 and Grade 3 tumors.

lines have demonstrated the significant growth-inhibitory effects of various means of NTSR1 blockage. Earlier studies using SR48692, the first NTSR1 antagonist has shown a significant reduction of cancer cell proliferation in ex vivo cell culture or xenografted model $[18,19]$. In the study of Souaze et al., small interfering RNA knockdown of NTSR1 in xenografted MDA-MB-231 cells resulted in a $70 \%$ decrease in tumor growth as compared to wild-type cells [5]. In the study of Heakal et al, nanoliposomal short-chain ceramide, a chemical that can inhibit agonist-dependent translocation of NTSR1, was found to significantly inhibit NTSR1-mediated MDAMB-231 breast cancer progression (mitogenesis, migration, and matrix metalloproteinase activity) [8]. Castillo-Rodriguez et al. recently developed a neurotensinpolyplex that transfects therapeutic genes into NTSR1expressing breast cancer cells (MDA-MB-231 cells) and kills tumor cells in both in vitro and in vivo models [20]. Unfortunately, to the best knowledge of the authors, a clinically usable NTSR1 antagonist is still not available. The exact efficacy of the proposed anti-NTSR 1 treatment awaits validation through future study, provided a clinically applicable drug becomes a reality in the near future with the recent better understanding of the chemical structure of NTSR1 [21].

\section{Acknowledgements}

This study was supported by the Calgary Laboratory Services (CLS, RS10-534). Ms. Michelle Darago provided very helpful technical support.

\section{REFERENCES}

[1] R. Carraway and S. E. Leeman, "The Isolation of a New Hypotensive Peptide, Neurotensin, from Bovine Hypo- thalami," The Journal of Biological Chemistry, Vol. 248, No. 19, 1973, pp. 6854-6861.

[2] R. Carraway and S. E. Leeman, "Characterization of Radioimmunoassayable Neurotensin in the Rat. Its Differential Distribution in the Central Nervous System, Small Intestine, and Stomach," The Journal of Biological Chemistry, Vol. 251, No. 22, 1976, pp. 7045-7052.

[3] C. F. Ferris, M. J. Armstrong, J. K. George, C. A. Stevens, R. E. Carraway and S. E. Leeman, "Alcohol and Fatty Acid Stimulation of Neurotensin Release from Rat Small Intestine," Endocrinology, Vol. 116, No. 3, 1985, pp. 11331138. doi:10.1210/endo-116-3-1133

[4] B. M. Evers, "Neurotensin and Growth of Normal and Neoplastic Tissues," Peptides, Vol. 27, No. 10, 2006, pp. 2424-2433. doi:10.1016/j.peptides.2006.01.028

[5] F. Souaze, S. Dupouy, V. Viardot-Foucault, E. Bruyneel, S. Attoub, C. Gespach, et al., "Expression of Neurotensin and NT1 Receptor in Human Breast Cancer: A Potential Role in Tumor Progression," Cancer Research, Vol. 66, No. 12, 2006, pp. 6243-6249. doi:10.1158/0008-5472.CAN-06-0450

[6] S. Somai, A. Gompel, W. Rostene and P. Forgez, "Neurotensin Counteracts Apoptosis in Breast Cancer Cells," Biochemical and Biophysical Research Communications, Vol. 295, No. 2, 2002, pp. 482-488.

doi:10.1016/S0006-291X(02)00703-9

[7] S. Dupouy, V. Viardot-Foucault, M. Alifano, F. Souaze, G. Plu-Bureau, M. Chaouat, et al., "The Neurotensin Receptor-1 Pathway Contributes to Human Ductal Breast Cancer Progression," PloS One, Vol. 4, No. 1, 2009, Article ID: e4223.

[8] Y. Heakal and M. Kester, "Nanoliposomal Short-Chain Ceramide Inhibits Agonist-Dependent Translocation of Neurotensin Receptor 1 to Structured Membrane Microdomains in Breast Cancer Cells," Molecular Cancer Research, Vol. 7, No. 5, 2009, pp. 724-734. doi:10.1158/1541-7786.MCR-08-0322

[9] D. Gully, B. Labeeuw, R. Boigegrain, F. Oury-Donat, A. Bachy, M. Poncelet, et al., "Biochemical and Pharmacological Activities of SR 142948A, a New Potent Neurotensin Receptor Antagonist," Journal of Pharmacology and Experimental Therapeutics, Vol. 280, No. 2, 1997, pp. 802-812.

[10] D. Zhao, Y. Zhan, H. Zeng, H. W. Koon, M. P. Moyer and C. Pothoulakis, "Neurotensin Stimulates Expression of Early Growth Response Gene-1 and EGF Receptor through MAP Kinase Activation in Human Colonic Epithelial Cells," International Journal of Cancer, Vol. 120, No. 8, 2007, pp. 1652-1656. doi:10.1002/ijc.22407

[11] D. Zhao, Y. Zhan, H. W. Koon, H. Zeng, S. Keates, M. P. Moyer and C. Pothoulakis, "Metalloproteinase-Dependent Transforming Growth Factor-Alpha Release Mediates Neurotensin-Stimulated MAP Kinase Activation in $\mathrm{Hu}-$ man Colonic Epithelial Cells," The Journal of Biological Chemistry, Vol. 279, No. 42, 2004, pp. 43547-43554. doi:10.1074/jbc.M401453200

[12] S. Hassan, P. R. Dobner and R. E. Carraway, "Involvement of MAP-Kinase, PI3-Kinase and EGF-Receptor in 
the Stimulatory Effect of Neurotensin on DNA Synthesis in PC3 Cells," Regulatory Peptides, Vol. 120, No. 1-3, 2004, pp. 155-166. doi:10.1016/j.regpep.2004.03.004

[13] M. J. Alexander and S. E. Leeman, "Estrogen-Inducible Neurotensin Immunoreactivity in the Preoptic Area of the Female Rat," Journal of Comparative Neurology, Vol. 345, No. 4, 1994, pp. 496-509. doi:10.1002/cne. 903450403

[14] J. J. Watters and D. M. Dorsa, "Transcriptional Effects of Estrogen on Neuronal Neurotensin Gene Expression Involve cAMP/Protein Kinase A-Dependent Signaling Mechanisms," The Journal of Neuroscience, Vol. 18, No. 17, 1998, pp. 6672-6680.

[15] W. H. Rostene and M. J. Alexander, "Neurotensin and Neuroendocrine Regulation," Frontiers in Neuroendocrinology, Vol. 18, No. 2, 1997, pp. 115-173. doi:10.1006/frne.1996.0146

[16] M. J. Alexander, "Colocalization of Neurotensin Messenger Ribonucleic Acid (mRNA) and Progesterone Receptor mRNA in Rat Arcuate Neurons under Estrogen-Stimulated Conditions," Endocrinology, Vol. 140, No. 11, 1999, pp. 4995-5003. doi:10.1210/en.140.11.4995

[17] P. Taneja, D. Maglic, F. Kai, S. Zhu, R. D. Kendig, E. A.
Fry and K. Inoue, "Classical and Novel Prognostic Markers for Breast Cancer and Their Clinical Significance," Clinical Medicine Insights: Oncology, Vol. 4, 2010, pp. 15-34.

[18] K. Iwase, B. M. Evers, M. R. Hellmich, H. J. Kim, S. Higashide, D. Gully, et al., "Indirect Inhibitory Effect of a Neurotensin Receptor on Human Colon Cancer (LoVo) Growth," Surgical Oncology, Vol. 5, No. 5-6, 1996, 245251. doi:10.1016/S0960-7404(96)80028-4

[19] T. W. Moody, J. Chiles, M. Gasibang, E. Moody, D. Chan and T. P. Davis, "SR48692 Is a Neurotensin Receptor Antagonist Which Inhibits the Growth of Small Cell Lung Cancer Cells," Peptides, Vol. 22, No. 1, 2001, pp. 109-115. doi:10.1016/S0196-9781(00)00362-4

[20] R. Castillo-Rodriguez, A. Arango-Rodriguez, L. Escobedo, H. Rubio-Zapata, V. Tellez-Lopez, T. Mejia-Castillo, et al., "Neurotensin-Polyplex as a Potential Tool in Gene Therapy for Human Breast Cancer," European Journal of Cancer, Vol. 48, Suppl. 1, 2012, p. S93. doi:10.1016/S0959-8049(12)70252-6

[21] J. F. White, N. Noinaj, Y. Shibata, J. Love, B. Kloss, F. $\mathrm{Xu}$, et al., "Structure of the Agonist-Bound Neurotensin Receptor," Nature, Vol. 490, No. 7421, 2012, pp. 508513. doi: $10.1038 /$ nature 11558 